\title{
Gene therapy is not eugenics
}

Sir - Your excellent editorial ${ }^{1}$ provided a thoughtful and informative overview of the escalating concerns regarding the role of genomics in a possible eugenicist agenda in China and elsewhere. While the main point of this editorial is well-taken, lumping potential corrective genetic therapies with eugenics is ill-advised. Although advancing genetic technology will likely pose new dilemmas for researchers and society as a whole, as illustrated in recent Chinese laws, a distinction must be made between corrective genetic therapies, like gene therapy, and molecular diagnostics. The editorial lumps together corrective genetic therapies with molecular diagnostics by stating that the intent of genetics research is eugenicist by implication. While it is true that molecular diagnostics are capable of accomplishing an eugenicist agenda ${ }^{2}$, the same cannot be said of corrective genetic therapies.

Somatic gene therapy should not be considered a potential tool of eugenics; rather, it is a natural evolution in pharmaceutical technology. Clearly, germline gene therapy poses significantly different ethical questions ${ }^{3}$, but somatic gene therapy can be considered as a development of 'DNA drugs'4. Important differences exist between traditional drug development and gene therapy development, but the nature of gene therapeutic agents (GTAs) is similar to traditional pharma- ceuticals. One difference between pharmaceutical and GTA development is that a pharmaceutical agent is often a single chemical entity that has undergone extensive preclinical investigation, while potential GTAs are numerous even for a particular genetic disease like cystic fibrosis $(C F)^{5}$. Another important difference between traditional pharmaceutics and GTAs is that a better understanding of the molecular biology of gene transfer agents directly impacts the outcome of GTA development ${ }^{6}$. But the overall similarity between most traditional pharmaceutics and GTAs is that both attempt to circumvent or abrogate a disease process by intervening in the pathophysiologic cascade that leads to a particular disease. In $\mathrm{CF}$, for example, traditional pharmaceutical development led to the use of human recombinant DNase for the treatment of the abnormally thick lung secretions which afflict CF patients. The increased amount of bacterial and neutrophilic DNA is a consequence of mutant cystic fibrosis transmembrane regulator (CFTR); cleavage of this DNA into smaller pieces reduces the viscosity of lung secretions, and allows easier clearance by patient ${ }^{7}$. The principle behind CF gene therapy is similar and involves complementation or augmentation of the mutant CFTR with expression of wild-type CFTR which restores normal physiology. Both drugs interfere with the pathophysiologic cascade that leads to the manifestations of CF, but they affect different steps in that cascade. Gene therapy as a drug cannot be considered part of a eugenicist agenda because the intention of such agents is to help patients with disease traits to live longer, healthier, more productive lives.

Corrective genetic therapies should be considered the antithesis of eugenics because the aim of gene therapy, like other treatments of inherited disease, is to allow patients with genetic diseases to live longer, rather than to eliminate certain genetic traits from the human gene pool. This should not distract attention from the important dilemmas posed by advances in other genomics technology discussed in the editorial, but rather should serve to highlight the role of somatic gene therapy as a natural extension of current treatments of inherited disease.

\section{John A. Wagner}

Department of Molecular Pharmacology Stanford University Schoool of Medicine, Stanford, CA 945305-5332, USA. e-mail: wagner@leland.stanford.edu

1. Editorial. Nature Genet. 15, 1-2 (1997)

2. Albin, R.L. Nature Genet 14, 17 (1996)

3. McGleenan, T. J. Med. Ethics 21, 350-355 (1995).

4. Crystal, R.G. Nature Med. 1, 15-17 (1995).

5. Wagner, J.A. \& Gardner, P. Annu. Rev. Med. (in press).

6. Wilson, J.M. J. Clin. Invest. 97, 1138-1141 (1996).

7. Harris, C.E. \& Wilmott, R.W. Curr. Opin. Pediatr. 6 , 234-238(1994).

\section{Putting a hold on ' $H L A-H$ '}

Sir - Hereditary haemochromatosis $(\mathrm{HH})$ is one of the most common human genetic diseases, characterized by a massive iron overload. Estimated frequencies vary from 1 in 200 in Brittany to 1 in 1,000 in other white populations ${ }^{1}$. The haemochromatosis gene was mapped 20 years ago by linkage disequilibrium with the HLA-A locus on chromosome 6. Last year, Feder et al. ${ }^{2}$ reported in Nature Genetics the identification of the putative $\mathrm{HH}$ gene, and identified a missense mutation, $\mathrm{C} 282 \mathrm{Y}$, present in homozygous form in $83 \%$ patients. Two other groups have reported even higher frequencies in their populations $s^{3,4}$.

The $\mathrm{HH}$ candidate gene is located 4.5 megabases $(\mathrm{Mb})$ telomeric to $H L A-A$, and due to its similarity to HLA class I genes, Feder $e t$ al. named it ' $H L A-H$ '. However, this designation had already been used in 1990 by Chorney et al. ${ }^{5}$, who described a pseudogene located between $H L A-B$ and $H L A-A, 0.36 \mathrm{Mb}$ centromeric to HLA-A (see Fig. 1). Comparison of the original HLA-H pseudogene (GenBank \#M31944) and the haemochromatosis gene (GenBank \#U60319) confirms that the two are different.

We suggest that to avoid any further confusion, the haemochromatosis gene should be renamed. The accepted representation of the haemochromatosis locus in the Genome Database (symbols approved by the HUGO./GDB Nomenclature Committee) is $H F E$.

\section{Bernard Mercier, Catherine Mura \& Claude Ferec}

Centre de Biogenetique, ETSBO-UBO-CHUBP454, Brest 29275, France

1. Meyer, T. et al. J. Intern. Med. 227, 397 (1990).

2. Feder, J.N. et al. Nature Genet. 13,399-408 (1996).

3. Jazwinska, E.C. et al. Nature Genet. 14, 249-251 (1996).

4. Jouanolle, A.M. et al. Nature Genet. 14, 251-252 (1996).

5. Chorney, M.J. et al. Mol. Cell. Biol. 10, 243-253 (1990).

Sir - The naming of HLA class I and II genes of the HLA region is the responsibility of the WHO Nomenclature Committee for Factors of the HLA System ${ }^{1}$. Furthermore, the use of the designation ' $H L A$ ' is reserved for the class I and II genes named by this committee. Included within the family of HLA class I heavy genes are several pseudogenes of which one, $H L A-H$, was named in 1990 (ref. 2). Recently, Feder 